\title{
Composition, variation, expression and evolution of low-molecular-weight glutenin subunit genes in Triticum urartu
}

Guangbin Luo ${ }^{1,2}$, Xiaofei Zhang ${ }^{1,4}$, Yanlin Zhang ${ }^{3}$, Wenlong Yang ${ }^{1}$, Yiwen Li ${ }^{1}$, Jiazhu Sun ${ }^{1}$, Kehui Zhan ${ }^{3}$, Aimin Zhang ${ }^{1,3^{*}}$ and Dongcheng Liu ${ }^{1 *}$

\begin{abstract}
Background: Wheat (AABBDD, $2 n=6 x=42$ ) is a major dietary component for many populations across the world. Bread-making quality of wheat is mainly determined by glutenin subunits, but it remains challenging to elucidate the composition and variation of low-molecular-weight glutenin subunits (LMW-GS) genes, the major components for glutenin subunits in hexaploid wheat. This problem, however, can be greatly simplified by characterizing the LMW-GS genes in Triticum urartu, the A-genome donor of hexaploid wheat. In the present study, we exploited the high-throughput molecular marker system, gene cloning, proteomic methods and molecular evolutionary genetic analysis to reveal the composition, variation, expression and evolution of LMW-GS genes in a T. urartu population from the Fertile Crescent region.
\end{abstract}

Results: Eight LMW-GS genes, including four m-type, one s-type and three i-type, were characterized in the T. urartu population. Six or seven genes, the highest number at the Glu-A3 locus, were detected in each accession. Three i-type genes, each containing more than six allelic variants, were tightly linked because of their co-segregation in every accession. Only 2-3 allelic variants were detected for each $\mathrm{m}$ - and s-type gene. The m-type gene, TuA3-385, for which homologs were previously characterized only at Glu-D3 locus in common wheat and Aegilops tauschii, was detected at Glu-A3 locus in T. urartu. TuA3-460 was the first s-type gene identified at Glu-A3 locus. Proteomic analysis showed 1-4 genes, mainly i-type, expressed in individual accessions. About $62 \%$ accessions had three active i-type genes, rather than one or two in common wheat. Southeastern Turkey might be the center of origin and diversity for T. urartu due to its abundance of LMW-GS genes/genotypes. Phylogenetic reconstruction demonstrated that the characterized T. urartu might be the direct donor of the Glu-A3 locus in common wheat varieties.

Conclusions: Compared with the Glu-A3 locus in common wheat, a large number of highly diverse LMW-GS genes and active genes were characterized in $T$. urartu, demonstrating that this progenitor might provide valuable genetic resources for LMW-GS genes to improve the quality of common wheat. The phylogenetic analysis provided molecular evidence and confirmed that T. urartu was the A-genome donor of hexaploid wheat.

Keywords: Low-molecular-weight glutenin subunits, Triticum urartu, Glu-A3, Proteomics, Evolution

\footnotetext{
* Correspondence: amzhang@genetics.ac.cn; dcliu@genetics.ac.cn

'State Key Laboratory of Plant Cell and Chromosome Engineering, National

Center for Plant Gene Research, Institute of Genetics and Developmental

Biology, Chinese Academy of Sciences, 1 West Beichen Road, Chaoyang

District, Beijing 100101, China

Full list of author information is available at the end of the article
} 


\section{Background}

Wheat flour can be made into a wide variety of foods due to the unique viscoelastic properties of dough $[1,2]$. These viscoelastic properties result from gluten proteins, which account for about $80 \%$ of the total grain proteins $[3,4]$. Wheat gluten is composed of two main components: glutenin and gliadin. Glutenin plays a major role in dough's elasticity, while gliadin contributes mainly to dough's viscosity [5]. According to their relative mobility in sodium dodecyl sulphate polyacrylamide gel electrophoresis (SDS-PAGE), glutenin proteins are generally divided into high-molecular-weight glutenin subunits (HMW-GSs) and low-molecular-weight glutenin subunits (LMW-GSs) [6]. LMW-GSs have molecular weights ranging from $20 \mathrm{kDa}$ to $45 \mathrm{kDa}$, making up $60 \%$ of glutenin proteins and one third of seed storage proteins [3,7]. Based on the first amino acid of the mature proteins, LMW-GSs have been classified into three types: i- (isoleucine), $\mathrm{m}$ - (methionine) and s- (serine) [8].

LMW-GSs are encoded by a multi-gene family whose members are located at Glu-A3, Glu-B3 and Glu-D3 loci on the short arms of homologous chromosomes $1 \mathrm{~A}, 1 \mathrm{~B}$, and $1 \mathrm{D}$, respectively [9]. Without a complete genome sequence, it is hard to determine the exact members of LMW-GS gene family in a wheat variety. In the past decade, the LMW-GS gene family members were characterized in only a few wheat varieties, including Norin 61, Glenlea and Xiaoyan 54 [10-12]. Twelve to 19 LMWGS genes were identified from individual varieties using complementary methods, including cDNA or DNA BAC library screening and proteomic analysis. Recently, a new molecular marker system was developed to identify LMW-GS gene family members which used highresolution capillary electrophoresis to separate fragments of gene members with three conserved primer sets (LMWGS1, LMWGS2 and LMWGS3) [13]. Using this marker system, more than 15 LMW-GS genes were detected from single wheat variety [13]. This marker system was also used as a complementary tool for the allelic determination of LMW-GS genes at Glu-B3 locus in wheat cultivars and segregating populations [14]. A full-length gene-cloning method based on this marker system has been used to clone 16 or 17 LMWGS genes in individual bread wheat genotypes [15]. Both the marker system and the gene cloning method were applied to investigate the composition of LMWGS genes in large populations, including Aroona nearisogenic lines and the micro-core collections (MCC) of Chinese wheat germplasm $[16,17]$, demonstrating their efficiency in dissecting this complex gene family in common wheat.

Wild progenitors and relatives could provide tremendous genetic variability to broaden the gene-pool of common wheat [18]. In the past decades, several important agronomic genes have been well characterized, such as the stem rust resistance gene $\mathrm{Sr} 47$, the leaf rust resistance genes ( $L r 41, L r 42$ and $L r 43$ ) from Aegilops tauschii, the high grain protein content (Gpc-B1) gene from tetraploid wheat and the chromosome arm 1RS containing both disease resistance and high yield genes from rye [19-22]. T. urartu is the wild diploid wheat from the Fertile Crescent region, and has long been considered as the A-genome donor in polyploid wheat species [23]. Isozyme, RAPD and AFLP markers have detected large genetic variations in T. urartu populations $[24,25]$. Recently, a set of genes were also characterized in $T$. urartu, e.g., the powdery mildew resistance gene $(P m U)$, and the grain length controlling gene (TuGASR7) [26-28]. Abundant variability of storage proteins in $T$. urartu, was detected in gliadin proteins and HMW-GSs using electrophoretic procedures or nucleotide sequence analysis $[29,30]$. Several variants with repetitive domain length polymorphism were also observed in LMW-GS genes [31]. However, the detailed composition and genetic diversity of LMW-GS genes in T. urartu remain unknown.

Dissecting the composition and diversity of LMW-GS genes in T. urartu is prerequisite to broadening the genetic resources for bread-making quality improvement in common wheat; unraveling the genetic diversity of $T$. urartu will facilitate its gene and germplasm conservation. In this study, a systematic molecular analysis of LMW-GS genes in T. urartu was conducted using complementary approaches, including highthroughput molecular marker system, gene cloning, two-dimensional electrophoresis (2-DE), liquid chromatography tandem mass spectrometry (LC-MS/MS), matrix assisted laser desorption/ionization time of flight tandem mass spectrometry (MALDI-TOF/TOF-MS) and SDSPAGE. The gene composition, variation, organization and expression pattern were extensively investigated in 157 accessions collected from the Fertile Crescent region, which is widely considered as the center of origin and diversity of T. urartu [25,32]. Genetic diversity of LMW-GS genes and genotypes in T. urartu and their evolutionary clues pertaining to wheat species of different ploidy were further discussed.

\section{Results}

Composition and variation of LMW-GS genes in T. urartu

For each conserved primer set of the LMW-GS marker system [13], more than 16 DNA fragments were amplified from the T. urartu population. Totally, 25 non-redundant DNA fragments from the population, with six or seven from each accession, were determined and named according to the experimental or theoretical size of their corresponding fragments amplified by the LMWGS1 primer set (Table 1) [13,15,17]. Typically, the sequenced accession, 
Table 1 LMW-GS genes and their allelic variants identified in T. urartu population using the LMW-GS gene molecular marker system

\begin{tabular}{|c|c|c|c|c|c|c|c|c|c|c|c|c|c|c|c|c|}
\hline \multirow{2}{*}{$\frac{\text { Gene }}{\text { TuA3-385 }}$} & \multicolumn{4}{|c|}{ Allelic variants $^{\mathrm{a}}$} & \multicolumn{4}{|c|}{ LMWGS1 $^{\text {b }}$} & \multicolumn{4}{|c|}{ LMWGS2 $^{\text {b }}$} & \multicolumn{4}{|c|}{ LMWGS3 $^{\text {b }}$} \\
\hline & TuA3-385 & & & & 385 & & & & 492 & & & & 383 & & & \\
\hline TuA3-391 & TuA3-373 & TuA3-391 & TuA3-392 & & 373 & 391 & 392 & & 480 & 484 & 501 & & 371 & 375 & 390 & \\
\hline TuA3-397 & TuA3-397 & & & & 397 & & & & 504 & & & & 396 & & & \\
\hline TuA3-400 & TuA3-400 & TuA3-402 & & & 400 & 402 & & & 506 & 509 & & & 399 & 402 & & \\
\hline TuA3-400 & TuA3-460 & TuA3-463 & TuA3-474 & & 460 & $N^{c}$ & 474 & & 566 & 569 & 580 & & 464 & 467 & 479 & \\
\hline \multirow[t]{2}{*}{ TuA3-502 } & TuA3-495 & TuA3-498 & TuA3-502 & TuA3-520 & $N$ & $N$ & $\mathrm{~N}$ & $N$ & 603 & $N$ & $\mathrm{~N}$ & $\mathrm{~N}$ & 532 & 535 & 538 & 561 \\
\hline & & TuA3-590 & TuA3-593 & & & $N$ & $N$ & & & $N$ & N & & & 633 & 636 & \\
\hline TuA3-538 & TuA3-535 & TuA3-538 & TuA3-657 & & 535 & 538 & 657 & & 641 & 644 & 753 & & 574 & 577 & 697 & \\
\hline \multirow[t]{2}{*}{ TuA3-576 } & TuA3-406 & TuA3-555 & TuA3-576 & TuA3-579 & $N$ & 555 & 576 & $N$ & 517 & $N$ & 682 & 685 & 443 & 597 & 618 & 621 \\
\hline & & TuA3-597 & TuA3-669 & & & 597 & 669 & & & $N$ & 773 & & & 640 & 720 & \\
\hline
\end{tabular}

${ }^{\mathrm{a}} \mathrm{A}$ single gene could be detected by no less than one primer set, and the correspondence among fragments detected by these three primer sets was established by their theoretical sizes. LMW-GS genes and allelic variants were named in accordance with the sizes of their corresponding fragments amplified by the LMWGS1 primer set practically or theoretically, and the major allelic variant was designated as the gene whereas the remainders as its allelic variants [17].

${ }^{\mathrm{b}}$ Three primer sets of the LMW-GS gene molecular marker system [13].

${ }^{\mathrm{c}}$ Not amplified by the specific primer sets.

PI428198 (G1812) [28], had seven LMW-GS genes, including TuA3-385, ТuA3-392, TuA3-397, TuA3-402, TuA3-520, TuA3-538 and TuA3-576 (Figure 1; Table 1). Among 157 accessions, 15 different genotypes (U1-U15) were identified; each genotype had unique fragment sizes except for U5 and U6, which were discriminated by SNPs within three LMW-GS genes (TuA3-502, TuA3-538 and TuA3-576) according to the subsequent gene cloning data (Table 2). Regarding the frequencies of the genotypes in the T. urartu population, U6 was the most abundant (39 accessions), followed by U2 (35 accessions), U10 (21 accessions) and U8 (16 accessions); the remaining 11 genotypes totally accounted for $24 \%$ of accessions, in which the genotypes U1, U7, U11 and U12 were discovered in only one or two accessions (Table 2).
To further characterize the LMW-GS genes represented by these DNA fragments, 50 typical accessions, covering all 15 genotypes, were subjected to gene cloning using the full-length gene cloning method (Additional file 1: Table S1) [15]. Generally, six or seven LMW-GS gene sequences were cloned in each accession, which matched well with six or seven DNA fragments detected with the marker system. Totally, 148 LMW-GS sequences were obtained and deposited in GenBank (KM065455-KM065457, KM085178-KM085322); these sequences were derived from eight LMW-GS genes (i.e., TuA3-385, TuA3-391, ТuA3-397, ТиA3-400, ТиА3-460, ТиА3-502, ТиА3-538 and TuA3-576) determined in the T. urartu population due to the redundancy and large number of allelic variants (Table 2). Among these genes, only two or three variants

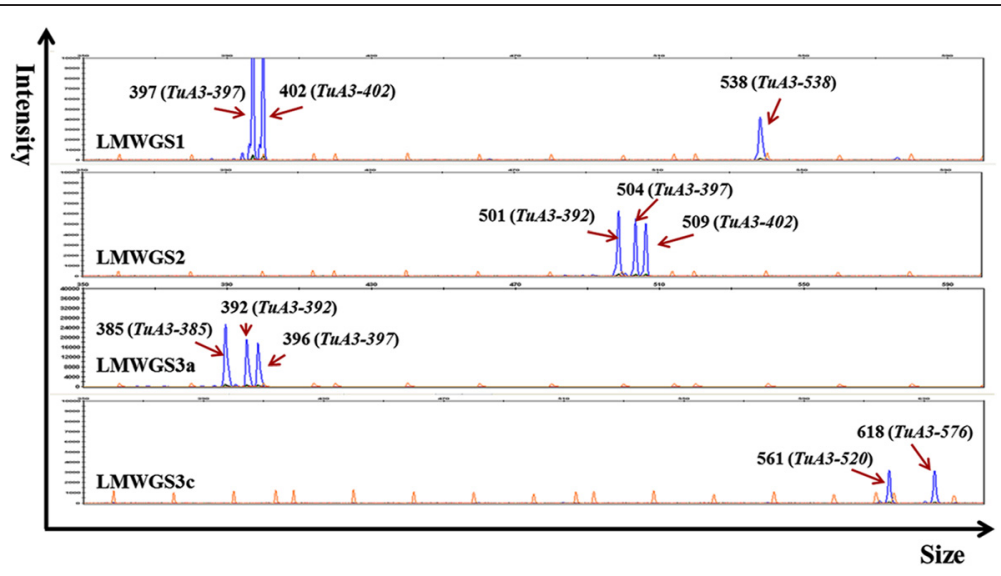

Figure 1 Electropherograms of DNA fragments detected in accession PI428198 using the LMW-GS gene molecular marker system. The horizontal axis shows the detected fragment sizes, and the vertical axis displays the signal intensities during the capillary electrophoresis. The orange peaks were size standard DNA fragments in the GeneScan 1200 LIZ and each blue peak represents a LMW-GS gene. 
Table 2 Genotypes and geographic distribution of LMW-GS genes in T. urartu population

\begin{tabular}{|c|c|c|c|c|c|c|c|c|c|c|c|c|c|c|c|}
\hline \multirow[t]{2}{*}{ Genotype } & \multicolumn{4}{|l|}{ m-type } & \multirow{2}{*}{$\begin{array}{l}\text { s-type } \\
\text { TuA3-460 }\end{array}$} & \multicolumn{3}{|l|}{ i-type } & \multicolumn{6}{|c|}{ Original region ${ }^{\#}$} & \multirow{2}{*}{$\begin{array}{l}\text { Accession } \\
\text { number }\end{array}$} \\
\hline & TuA3-385 & TuA3-391 & TuA3-397 & TuA3-400 & & TuA3-502 & TuA3-576 & TuA3-538 & Iraq & Iran & Armenia & Lebanon & Syria & Turkey & \\
\hline U1 & TuA3-385a ${ }^{+}$ & TuA3-373 & TuA3-397a & & & TuA3-502c & TuA3-576d & TuA3-538a* & & & & & & 1 & 1 \\
\hline U2 & TuA3-385b & TuA3-391 & TuA3-397a & & & TuA3-502b & TuA3-406 & TuA3-538c ${ }^{*}$ & 1 & 1 & & 9 & 1 & 23 & 35 \\
\hline U3 & TuA3-385a & TuA3-391 & TuA3-397a & & & TuA3-498* & TuA3-597* & TuA3-535* & & & & & & 5 & 5 \\
\hline U4 & TuA3-385b & TuA3-392 & TuA3-397a & & & TuA3-520* & TuA3-576 ${ }^{*}$ & TuA3-538d & & & & & & 7 & 7 \\
\hline U5 & TuA3-385a & TuA3-392 & TuA3-397a & & & TuA3-502C ${ }^{*}$ & TuA3-576b ${ }^{*}$ & TuA3-538b & & & & & & 9 & 9 \\
\hline U6 & TuA3-385a & TuA3-392 & TuA3-397a & & & TuA3-502a* & TuA3-576a & TuA3-538a* & & & & 36 & 2 & 1 & 39 \\
\hline U7 & TuA3-385a & TuA3-392 & TuA3-397a & & & TuA3-502d & TuA3-555 & TuA3-538a* & & & & 1 & & 1 & 2 \\
\hline U8 & TuA3-385a & TuA3-392 & TuA3-397b & & & TuA3-502a* & TuA3-579b* & TuA3-538b* & & & & 15 & 1 & & 16 \\
\hline U9 & TuA3-385a & TuA3-392 & TuA3-397a & & & TuA3-593 & TuA3-576b & TuA3-538e & & & & 1 & 1 & 4 & 6 \\
\hline U10 & TuA3-385a & TuA3-373 & TuA3-397a & & TuA3-460 & TuA3-502a* & TuA3-579a* & TuA3-538b & & & 1 & 19 & & 1 & 21 \\
\hline U11 & TuA3-385a & TuA3-373 & TuA3-397a & & TuA3-463 & TuA3-520* & TuA3-576 ${ }^{*}$ & TuA3-538a* & & & & & & 2 & 2 \\
\hline U12 & TuA3-385b & TuA3-373 & TuA3-397a & & TuA3-463 & TuA3-495 & TuA3-669 & TuA3-657 & & & & & & 1 & 1 \\
\hline U13 & TuA3-385a & TuA3-392 & TuA3-397a & & TuA3-474 & TuA3-502C & TuA3-576d & TuA3-538d & & & & 1 & & 3 & 4 \\
\hline U14 & TuA3-385a & TuA3-392 & TuA3-397a & TuA3-402 & & TuA3-520* & TuA3-576 ${ }^{*}$ & TuA3-538a* & & & & & & 5 & 5 \\
\hline U15 & & TuA3-392 & TuA3-397b ${ }^{*}$ & TuA3-400* & & TuA3-590 & TuA3-576e & TuA3-538a* & & & 4 & & & & 4 \\
\hline
\end{tabular}

"Letter following the number is used to distinguish allelic variants with the same size of DNA fragment.

"Asterisks label active genes with their protein products detected in SDS-PAGE or 2-DE.

"Lebanon and Turkey represent Northeastern Lebanon and Southeastern Turkey, respectively. 
were detected for each of the TuA3-385, TuA3-391, TuA3397, Tu-A3-400 and TuA3-460 genes. In contrast, at least seven variants were identified for each of the TuA3-502, TuA3-538 and TuA3-576 (Table 2). All allelic variants resulted in 15 genotypes at the Glu-A3 locus in T. urartu, which was consistent with the genotypes based on the size of DNA fragment in the marker system (Table 2).

\section{LMW-GS genes in T. urartu}

Among the eight genes, four (TuA3-385, TuA3-391, TuA3-397 and TuA3-400) were m-type, three (TuA3502, TuA3-538 and TuA3-576) were i-type and one (TuA3-460) was s-type.

\section{m-type LMW-GS genes}

TuA3-385 gene with two variants, TuA3-385a and $T u A 3-385 b$, was widely distributed in the $T$. urartu population. Both variants were supposed as pseudogenes due to immature stop codons at their repetitive domains (Additional file 1: Table S2; Additional file 2: Figure S1). Another common gene, TuA3-391 contained three allelic variants: TuA3-373, TuA3-391 and TuA3-392 (Additional file 1: Table S2; Additional file 2: Figure S2). All three allelic variants of TuA3-391 gene were pseudogenes because of immature stop codons at either their repetitive or C-terminal I domains. TuA3-397 was also a common gene in T. urartu population. TuA3-397a was the major allelic variant $(87.26 \%)$, but it might be a pseudo-gene. The other variant, TuA3-397b might encode an m-type LMW-GS for its intact open reading frame (ORF) (Additional file 1: Table S2; Additional file 2: Figure S3). TuA3-400 gene was seldom detected in T. urartu population; its two allelic variants (TuA3-400 and TuA3402) were only detected in four and five accessions, respectively (Additional file 1: Table S2). These two allelic variants shared $99 \%$ identity despite two 3-bp InDels and several SNPs (Additional file 2: Figure S3). The TuA3-402 allelic variant was a pseudo-gene due to the immature stop codon at its C-terminal II domain, whereas TuA3400 was supposed to be active for its intact ORF.

\section{i-type LMW-GS genes}

Three i-type genes: TuA3-502, TuA3-538 and TuA3-576 were identified in each $T$. urartu accession. The TuA3502 gene had nine variants: TuA3-495, TuA3-498, TuA3-502a/b/c/d, TuA3-520, TuA3-590 and TuA3-593 (Additional file 2: Figure S4). TuA3-502a was the major allelic variant of the TuA3-502 gene with an occupation of $48.41 \%$ accessions, and TuA3-502b was another widely distributed variant and detected in $22.29 \%$ accessions (Additional file 1: Table S2). Among nine variants, only TuA3-498, TuA3-502a and TuA3-502c might be active genes with intact ORFs.
Another i-type gene TuA3-538 had seven variants: TuA3-535, TuA3-538a/b/c/d/e and TuA3-657 in the T. urartu population. All the variants were supposed to be active genes for their intact ORFs, except for the TuA3$538 d$ variant. The $T u A 3-538 \mathrm{a} / \mathrm{b} / \mathrm{c} / \mathrm{d} / \mathrm{e}$ variants distinguished themselves mainly by different repeat number of CAG and CAA motifs at the C-terminal II domain in addition to several SNPs throughout their coding regions. The TuA3-535 variant shared $>99 \%$ identity with each of the $T u A 3-538 \mathrm{a} / \mathrm{b} / \mathrm{c} / \mathrm{d} / \mathrm{e}$ variants. Compared with the other allelic variants of the TuA3-538 gene, the long fragment of TuA3-657 was mainly derived from two insertions (24-bp and 87-bp) at the repetitive domain (Additional file 2: Figure S5). Among these allelic variants, the TuA3538a, TuA3-538b and TuA3-538c were widely distributed in the T. urartu population, occupying $85.35 \%$ of variants. TuA3-535, TuA3-538d, TuA3-538e and TuA3657 were rare, present in a few accessions (Additional file 1: Table S2).

The TuA3-576 gene contained 11 variants: TuA3-406, TuA3-555, TuA3-576a/b/c/d/e, TuA3-579a/b, TuA3-597 and TuA3-669. Many of them might be active genes based on their intact ORFs, whereas TuA3-406 and TuA3-576d were pseudo-genes with immature stop codons at their repetitive and C-terminal II domains. The TuA3-576a/b/c/d/e variants were distinguished by InDels at C-terminal II domain and SNPs throughout their coding sequences (Additional file 2: Figure S6). Long deletions and insertions caused different fragment lengths of the variants of TuA3-576 gene. Two deletions, 142-bp at the repetitive domain and 30-bp at the Cterminal I domain, were detected in the TuA3-406 variant. A 24-bp deletion was also found at the repetitive domain of TuA3-555 variant. In the TuA3-597 variant, a 24-bp insertion was identified at its repetitive domain. Three insertions were detected in the TuA3-669 variant: 33-bp and 69-bp at the repetitive domain, and 21-bp at the C-terminal III domain (Additional file 2: Figure S6). Among these variants, the TuA3-576a, TuA3-406, TuA3579a, TuA3-579b and TuA3-576b were widely distributed in the $T$. urartu population, with proportions of $24.84 \%, 22.29 \%, 13.38 \%, 10.19 \%$ and $9.55 \%$, respectively (Additional file 1: Table S2).

\section{s-type LMW-GS gene}

TuA3-460 has the N-terminal region (MENSHIPGLEKPS) of typical s-type LMW-GS and a short s-type protein specific peptide (TLSH) at the repetitive domain (Additional file 2: Figure S7). The first amino acid of the mature protein of TuA3-460 was Ser after the peptide MEN were cut from the original protein. Thus, TuA3-460 belonged to s-type LMW-GS. The TuA3-460 gene was the only stype LMW-GS gene detected in the T. urartu population. Its three variants: TuA3-460, TuA3-463 and TuA3-474, 
shared $>99 \%$ identity. And all were pseudo-genes with immature stop codons both at their repetitive and Cterminal I domains. Compared with TuA3-460, the 3-bp (CCA) and 12-bp (CAACAACAACAA) insertions at their repetitive domains were responsible for the larger fragment lengths of TuA3-463 and TuA3-474, respectively (Additional file 2: Figure S8). The TuA3-460 gene was detected in only $17.80 \%$ accessions, TuA3-460 (21 accessions), TuA3-463 (3 accessions), and TuA3-474 (4 accessions) (Additional file 1: Table S2).

\section{Expression of LMW-GS genes in T. urartu}

The bread-making quality of wheat flour is attributed greatly to the composition of LMW-GSs and the number of expressed genes $[12,16]$. To investigate the expression pattern of LMW-GS genes in T. urartu, four accessions from four genotypes, U2 (PI428202), U9 (PI428255), U10 (PI428270) and U8 (PI428335), in turn containing one, two, three and four genes with intact ORFs, were selected and subjected to proteomic analysis. All the spots on 2-DE gels of PI428202, PI428255 and PI428270, and the spots of LMW-GSs of PI428335 were identified by LC-MS/MS or MALDI-TOF/TOF MS (Figure 2).

Of the 25 spots investigated for PI428270 in the U10 genotype, three (spots 1, 2 and 3) were LMW-GSs, two were globulin, 13 were gliadins, and the remaining spots were other storage proteins (avenin, hordein and aveninlike precursor) (Figure 2a; Additional file 1: Table S3, S4 and S5). Spots 1, 2 and 3 were in turn matched to protein TuA3-538b, TuA3-579a and TuA3-502a in U10, and corresponded to the middle, upper and lower bands in SDS-PAGE due to their same mobility, respectively (Figure 2a; Additional file 1: Table S3). In the PI428335 accession with the U8 genotype, five spots were identified as LMW-GSs. Spots 1, 2 and 3 in turn matched deduced amino acid sequences of TuA3-538b, TuA3-579b and TuA3-502a, whereas both spots 4 and 5 matched TuA3397b. All of these spots had corresponding bands with the same mobility in SDS-PAGE (Figure 2b; Additional file 1: Table S3). Interestingly, TuA3-400, which was only identified in the U15 genotype, shared the same 2-DE protein spot and SDS-PAGE band with TuA3-397b due to their similar molecular mass and isoelectric point $(\mathrm{pI})$ value in our previous MS/MS identification (Data not shown) (Table 2; Figure 2e). In PI428202, spots 1 and 2 were proteins of the only active variant, TuA3-538c in U2; six (spot 1) and eight (spot 2) high-quality peptide sequences obtained by MS/MS analysis matched hypothetical polypeptides of TuA3-538c, respectively (Figure 2c; Additional file 1: Table S3). Moreover, these two spots also corresponded to the only band (TuA3-538c) detected with SDS-PAGE (Figure 2c). With regard to PI428255 of the U9 genotype, spot 1 was a protein product of the TuA3-
538 variant in U9 and corresponded to the lower band (TuA3-538e) in SDS-PAGE, and spot 2 was that of the TuA3-576b variant and matched the upper band (TuA3$576 \mathrm{~b}$ ) in SDS-PAGE (Figure 2d; Additional file 1: Table S3). The SDS-PAGE data for all the genotypes confirmed that four main types of bands corresponded to intact ORFs of TuA3-397/TuA3-400, TuA3-502, TuA3-538 and TuA3-576 in this $T$. urartu population by comparing their electrophoretic mobility with deduced protein molecular weights (Figure 2e). Collectively, the expression patterns of LMW-GS genes in T. urartu were consistent with the active genes determined using the LMW-GS marker system and full-length gene cloning method.

Generally, i-type genes were the main active genes in T. urartu, and one to three of them were expressed in individual accessions (Table 2, Figure 2e). One i-type variant, TuA3-538c, was expressed in 35 accessions of the U2 genotype. And all three i-type genes, TuA3-502, TuA3-538 and TuA3-576 were characterized as expressed genes in the U3, U5, U6, U10, U8, U11 and U14 genotypes, which together contained $61.78 \%$ of the total accessions (Table 2). All the m-type genes were pseudo-genes except for the TuA3-397b and TuA3-400 allelic variants, which were only detected in the U8 (TuA3-397b) and U15 (TuA3$397 b$ and TuA3-400) genotypes (Table 2, Figure 2e). None of the variants of the s-type gene, TuA3-460, were active as no protein bands were detected on 2-DE and SDSPAGE, which was consistent with the stop codons in their CDS regions (Table 2, Figure 2e).

\section{Characteristics of LMW-GS genes in T. urartu}

Based on the first amino acid of their mature protein sequences, the eight genes in $T$. urartu were classified into three types (m-, s- and i-). TuA3-385, TuA3-391, TuA3-397 and TuA3-400 were m-type, TuA3-460 was s-type and TuA3-502, TuA3-538 and TuA3-576 were i-type genes. Their deduced mature proteins contained three conserved domains (N-terminal domain, repetitive domain and C-terminal domain), except for the i-type subunit which lacked the $\mathrm{N}$-terminal domain (Additional file 2: Figure S9). Cysteine residues could form inter- and intra-chain disulphide bonds which are of great importance for the formation of glutenin polymers [3]. All the subunits identified in the T. urartu population contained eight cysteine residues. The location of these cysteine residues, in the $\mathrm{m}$ - and i-type genes, were conserved with six of the residues at the $\mathrm{C}$-terminal I domain and one at each of the C-terminal II and III domains, except for the first and the third cysteine residues in the m-type genes, TuA3-397b and TuA3-400 (Additional file 2: Figure S9). The m-type LMW-GSs were also different from the i-type genes in molecular weight. The estimated molecular weight of TuA3-397b and TuA3-400 were $31.77 \mathrm{kDa}$ and $31.90 \mathrm{kDa}$, respectively, substantially lower than the 


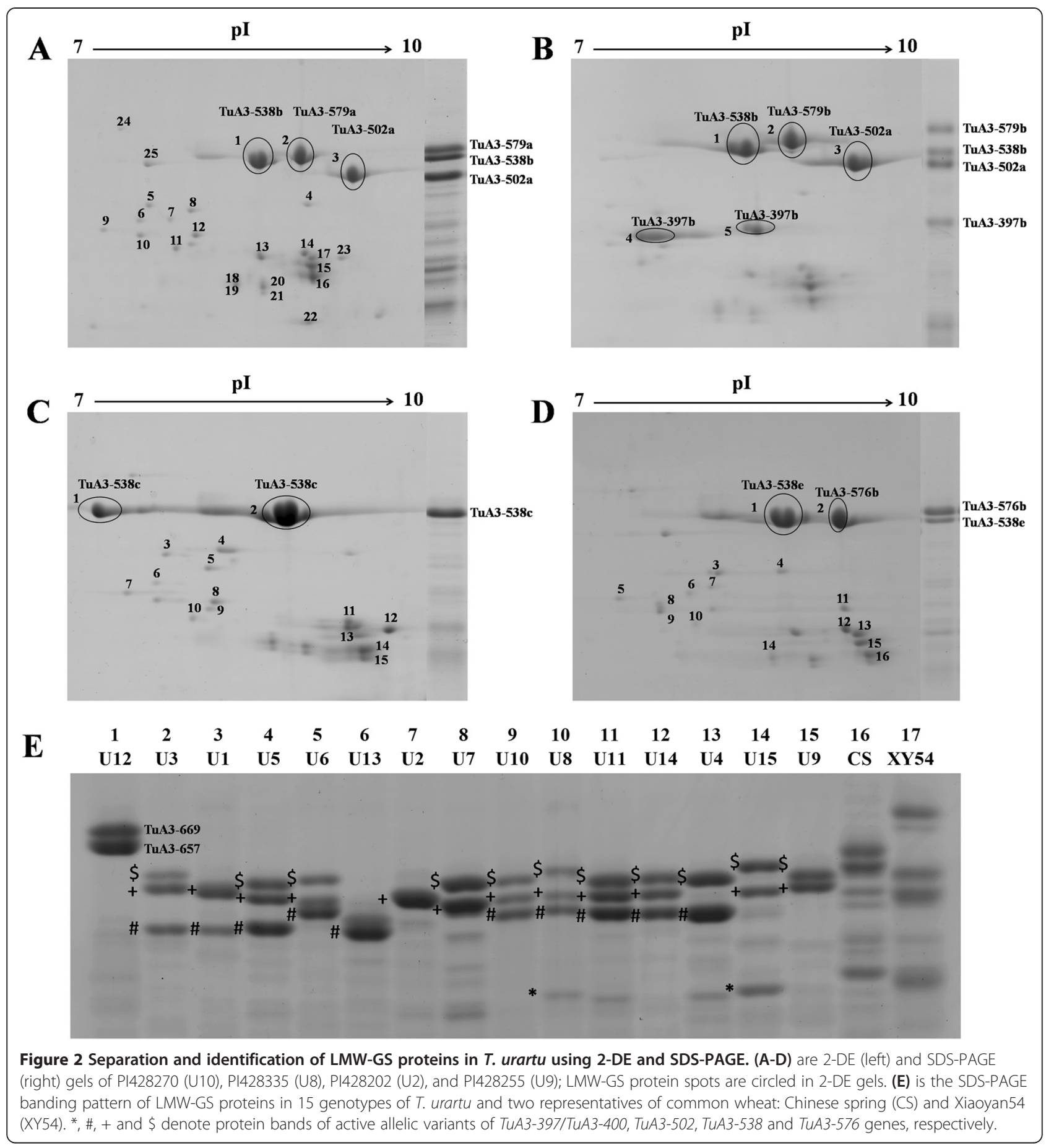

average molecular weight of all the i-type genes (TuA3502, $36.98 \mathrm{kDa}$; TuA3-538, $38.55 \mathrm{kDa}$; TuA3-576, $39.42 \mathrm{kDa}$ ) because of longer repetitive regions in the $\mathrm{i}$ type subunits (Additional file 2: Figure S9).

Among the three i-type genes, TuA3-502 was more tightly linked with TuA3-576 gene than TuA3-538 gene, since a set of variants of TuA3-502 and TuA3-576 genes co-segregated (e.g., TuA3-520 co-occurred with TuA3-
$576 c$ in U4, U11 and U14 genotypes, TuA3-590 was coupled with TuA3-576e in U15 genotype and TuA3$520 a$ co-occurred with TuA3-576a, TuA3-579a and TuA3-579b in U6, U10 and U8 genotypes, respectively.) (Table 2). Interestingly, the TuA3-502b, TuA3-406 and TuA3-538c variants might form a haplotype (TuA3502b/TuA3-406/TuA3-538c), because they co-segregated exclusively in 35 accessions of the U2 genotype (Table 2). 
TuA3-498, TuA3-535 and TuA3-597 might also form a haplotype due to their co-occurrence in the five accessions of the U3 genotype (Table 2).

All variants of the eight LMW-GS genes in T. urartu were subjected to phylogenetic analysis using ClustalW2 and MEGA 5. Two main clades were obtained in the phylogenetic tree, one containing all the $\mathrm{m}$ - and s-type genes, and the other including all the i-type genes (Figure 3). In the $\mathrm{m}$ - and s-type gene clade, four subclades were further divided, each containing variants of a single gene, except for the sub-clade of TuA3-397, where TuA3-400 was also involved (Figure 3). In the clade of i-type genes, three sub-clades were further divided, which corresponded to the TuA3-502, TuA3538 and TuA3-576 genes, accordingly (Figure 3).

\section{Geographic distribution of LMW-GS genes and genotypes in $T$. urartu}

The 157 analyzed T. urartu accessions were collected in the Fertile Crescent region, including northeastern Lebanon, southeastern Turkey, Armenia, Syria, Iraq and Iran, where many temperate-zone agricultural crops originated and were domesticated [33]. For the purpose of better exploitation and in situ genetic conservation of $T$. urartu germplasm, the geographic distribution of their LMW-GS genes/variants and genotypes was analyzed.

Southeastern Turkey was the region of the greatest diversity where all eight genes and 34 of their total 39 variants were detected, as well as ten unique variants were found (Table 2, Figure 4a). In northeastern Lebanon, 26 variants of seven genes (all except TuA3-400) were detected; all were shared by southeastern Turkey except TuA3-397b and TuA3-579b (Table 2, Figure 4a). With regard to the genotypes of LMW-GS genes, southeastern Turkey was also the region of the highest/(most abundant) diversity, as the majority of genotypes were detected there (Table 2, Figure 4b). Moreover, seven genotypes (U1, U3, U4, U5, U11, U12 and U14) were unique to southeastern Turkey (Table 2, Figure 4b). In northeastern Lebanon and Syria, seven and four genotypes were detected, respectively. All genotypes were also present in southeastern Turkey except for the U8 genotype (Table 2, Figure 4b). Despite containing the unique genotype, U15, Armenia shared the U10 genotype with southeastern Turkey and northeastern Lebanon (Table 2, Figure 4b). In Iraq and Iran, the only genotype, U2, was also detected in southeastern Turkey, northeastern Lebanon and Syria (Table 2, Figure 4b). In summary, southeastern Turkey should be the center of origin for T. urartu because the greatest diversity of LMW-GS genes/variants and genotypes were detected. And in the five remaining collection areas, almost all the genes/variants and genotypes were observed in southeastern Turkey.

\section{Discussion}

\section{LMW-GS genes in T. urartu}

Eight LMW-GS genes, i.e., four m-type, three i-type and one s-type genes, were characterized in the $T$. urartu

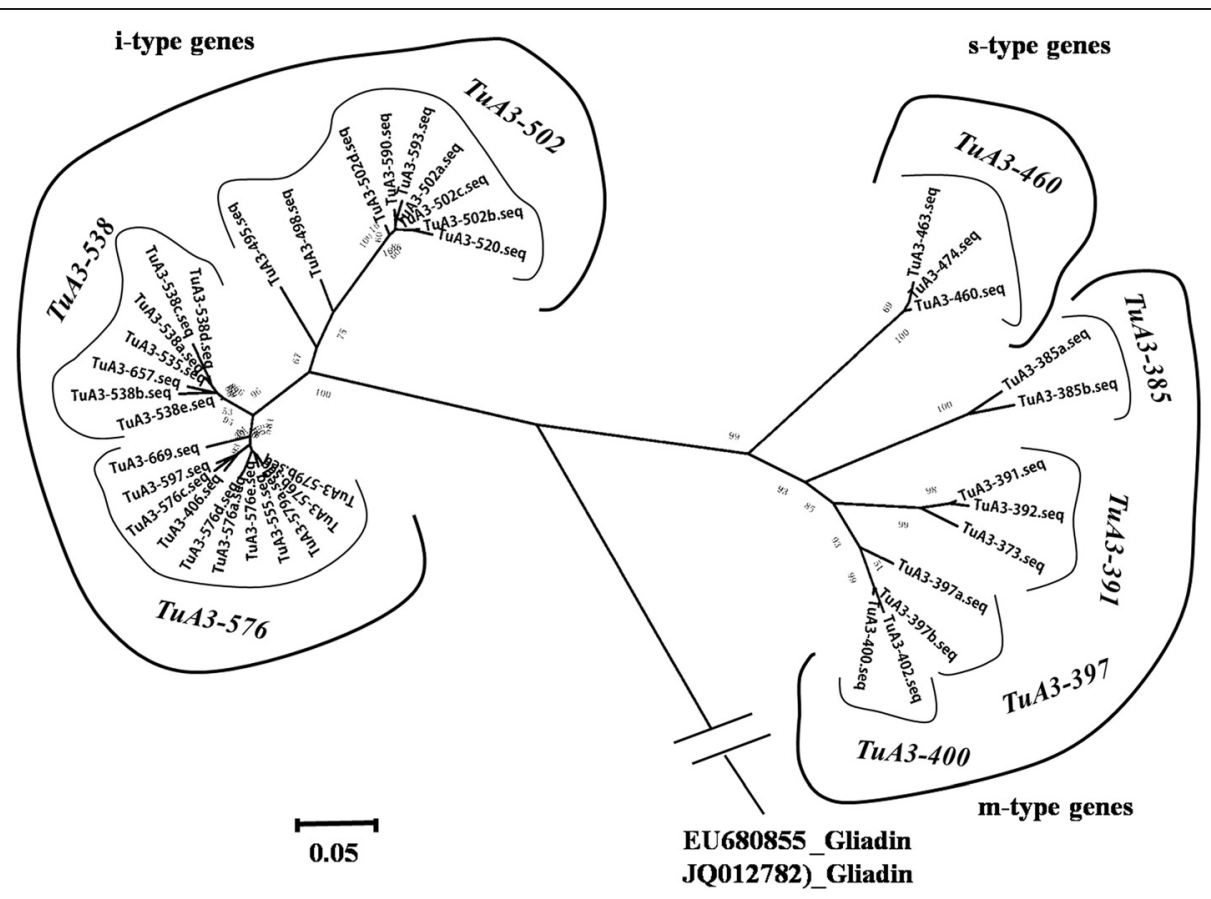

Figure 3 Phylogenetic reconstruction of all the LMW-GS genes and their allelic variants identified in the T. urartu population. All LMW-GS genes were divided into three groups, consistent with the i-, s- and m-type genes. 
A

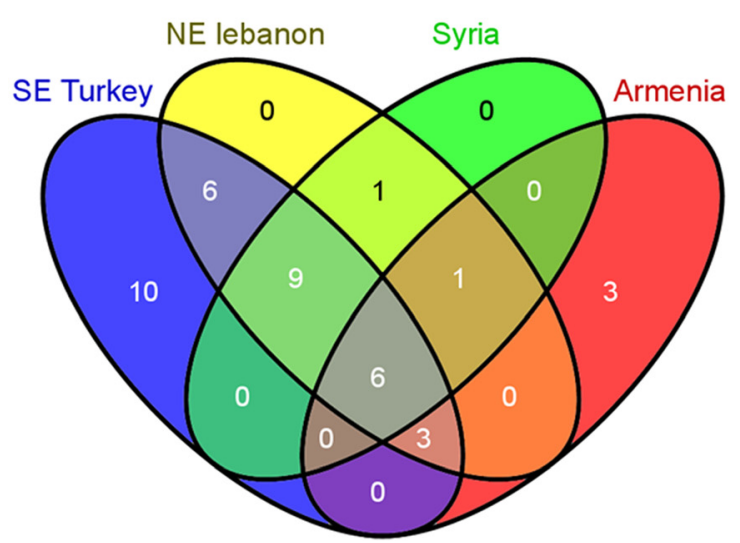

B

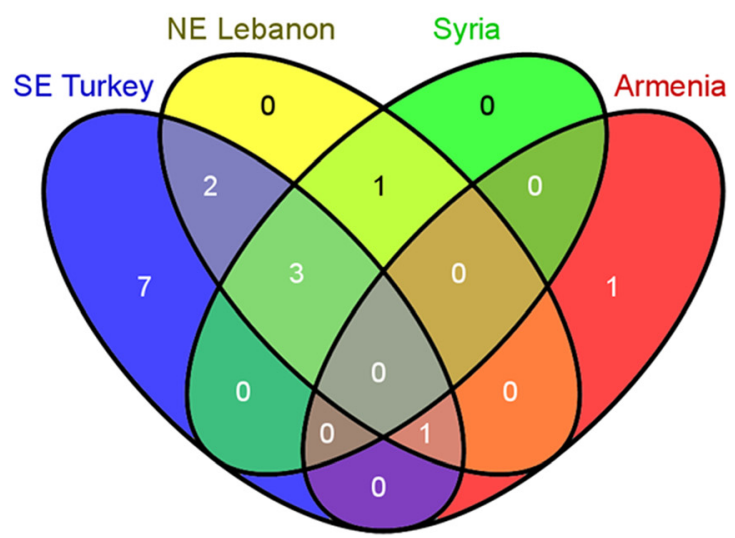

Figure 4 Geographic distribution of LMW-GS genes/allelic variants and genotypes in T. urartu. (A) Geographic distribution of LMW-GS genes/allelic variants. (B) Geographic distribution of LMW-GS genotypes. Iraq and Iran were not considered for only one accession was collected in each. SE Turkey stands for southeastern Turkey, and NE Lebanon for northeastern Lebanon.

population. In each accession, six to seven genes were detected, the highest number of LMW-GS genes reported at Glu-A3 locus to our best knowledge. To investigate the evolutionary relationships of LMW-GS genes between $T$. urartu and other diploid or polyploid wheats, all the gene sequences in $T$. urartu were queried with the nucleotide BLAST program in NCBI. Gene sequences sharing high identity (>90\%, even 99\%) with the LMW-GS genes in $T$. urartu were found (Additional file 1: Table S6).

As the homolog of TuA3-391 gene, A3-391 was previously identified in common wheat [17]. This gene was extremely conserved between $T$. urartu and common wheat with 99\% identity shared among its variants. (Additional file 1: Table S6). TuA3-397 was universal in T. urartu. Its variants, TuA3-397a and TuA3-397b, shared more than $96 \%$ identity with $A 3-394 \mathrm{~b}$ and $A 3-$ 400 , allelic variants of $A 3-400$ gene in common wheat, respectively (Additional file 1: Table S6) [17]. TuA3-397 and A3-400 should be homologs. Another gene, TuA3402 in T. urartu, also showed $99 \%$ identity with variants of A3-400 gene (Additional file 1: Table S6). TuA3-402 was only detected in U14 and U15 whereas TuA3-397 was a common gene in $T$. urartu. Moreover, both genes were located in the same branch in the phylogenetic tree (Figure 5a). It might be reasonable to hypothesize that the TuA3-402 gene was derived from a duplication of the TuA3-397 gene.

For TuA3-385, no homolog was found at Glu-A3 locus of other wheat species, but interestingly this gene shared $>98 \%$ identity with D3-385 at the Glu-D3 locus (e.g., JX878094) in hexaploid wheat [17] and the GluDt64 allele (EF437430) in Ae. tauschii (Additional file 1: Table S6). And phylogenetic analysis showed the TuA3385 and D3-385 genes clustered together (Figure 5a).
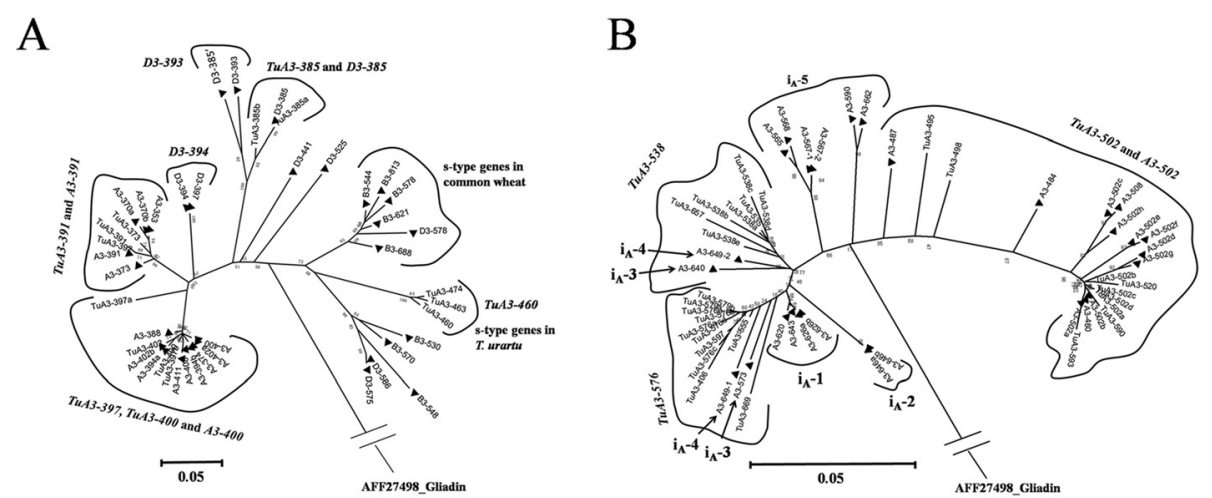

Figure 5 Phylogenetic analysis of deduced protein sequences of LMW-GS genes in T. urartu and common wheat. (A) Phylogenetic analysis of $\mathrm{m}$ - and s-type genes. (B) Phylogenetic analysis of i-type genes. Triangle represents sequences from common wheat. The gliadin protein sequence (AFF27498) was used as the out-group. 
Thus, the TuA3-385 and D3-385 genes were homologs between T. urartu and common wheat. However, TuA3385 was a pseudo-gene and D3-385 was an active gene in both common wheat and Ae. tauschii. This m-type gene should be an ancient gene that emerged before the divergence of the $\mathrm{A}$ and $\mathrm{D}$ genomes, but this gene was lost at the Glu-A3 locus during wheat polyploidization (Additional file 1: Table S6; Additional file 2: Figure S1). During evolution, this gene in T. urartu was mutated and became a pseudo-gene, but the gene in Ae. tauschii and common wheat maintained an intact ORF.

TuA3-460 was the first s-type LMW-GS gene detected at the Glu-A3 locus in Triticum. Interestingly, all of its BLAST hits $(\leq 90 \%$ identities) in BLAST databases were not s-type but m-type genes (Additional file 1: Table S6). And the phylogenetic analysis indicated that the variants of TuA3-460 were in the same clade with m-type genes at the Glu-B3 and Glu-D3 loci in Triticum aestivum (B3-530, B3-578, B3-570, D3-575 and D3-586) and Ae. speltoides (FJ824794) but not s-type genes (Figure $5 \mathrm{a}$ ). Protein sequence alignments were performed to understand the variations among TuA3-460, the known s- and m-type LMW-GSs (Additional file 2: Figure S7). Besides the conserved s-type N-terminal domain (MENSHIPGLEKPS) and the s-type specific peptide (TLSH) at the repetitive domain, TuA3-460 had 11 unique amino acids throughout the protein sequences, including one unique Cysteine residue at the $\mathrm{C}$-terminal II domain. Moreover, compared with the known m- and s-type LMW-GSs, TuA3-460 contained three deletions (PPFSQQ, PVLPQQ and PPFSQQQQ) at the repetitive domain (Additional file 2: Figure S7). Thus, TuA3-460 is a new s-type gene at the A genome, which is homologous with the s-type genes at the $\mathrm{B}$ and $\mathrm{D}$ genomes, rather than a chimeric gene containing s- and $\mathrm{m}$-type gene sequences. The s-type LMW-GS genes were closer to the m-type genes than the i-type ones (Figure 3). Our deduced protein sequence alignments also revealed that the s-type LMW-GSs had higher similarities with the m-type LMW-GSs (Additional file 2: Figure S9), and the m-type LMW-GSs shared the variations with all s-type proteins from $\mathrm{A}, \mathrm{B}$ and $\mathrm{D}$ genomes. Thus, $\mathrm{m}$-type gene should be the oldest type of LMW-GS gene, and s-type genes probably originated from m-type LMW-GS genes due to the mutation of MET to MEN in the N-terminal region, which was consistent with the previous observations $[3,17,34]$. Even though containing several unique features, TuA3-460 had a pretty high similarity with the m-type genes, especially possessed one insertion (KQLGQCSFQQPQQQ) at the $\mathrm{C}$-terminal domain and four amino acids (Additional file 2: Figure S9), which were exclusively contained in the m-type LMW-GSs. All these data indicate that this new s-type TuA3-460 gene also originated from the mtype LMW-GS genes. However, most features (specific amino acids and InDels) of the previously characterized s-type LMW-GSs could not be detected in TuA3-460, implying that TuA3-460 might not share the same evolutionary process with other s-type LMW-GS genes from the primitive m-type LMW-GS gene, or they could originate from different $\mathrm{m}$-type genes.

Three i-type genes, TuA3-502, TuA3-538 and TuA3576 detected in $T$. urartu were relatively conserved across Triticum species. All variants of the TuA3-502 gene share high identity ( $\geq 95 \%$ ) with the $A 3-502 b$ allele (JX877857) in common wheat, except for TuA3-498 and TuA3-593, which were homologous to A3-502f (JX878133) (93\% identity) and A3-484 (JX878099) (94\% identity), respectively (Additional file 1: Table S6. Many variants of the TuA3-538 gene showed higher identity ( $>97 \%$ ) with $A 3-$ 649-2 and $A 3-640$ than the other i-type genes in common wheat. The TuA3-576 gene in T. urartu might be the homolog of A3-649-1 and A3-573 in common wheat due to their high identity $(>97 \%)$. The variants of these three itype genes also showed high identities to i-type genes identified in wheat relatives, with TuA3-502c to AJ293098 (98\% identity) in Triticum durum, TuA3-538b to FJ441107 (95\% identity) in Triticum monococcum and TuA3-576a to DQ217661 (93\% identity) in Triticum dicoccoides (Additional file 1: Table S6).

However, the i-type genes preserved high polymorphisms at the Glu-A3 locus of Triticum [17]. In T. urartu, all accessions possessed three i-type genes, compared with 2-4 genes in common wheat, implying that the Glu-A3 locus might be derived from more than one origin of $T$. urartu and suffered rapid genome divergence. Moreover, all the three i-type genes in many $T$. urartu accessions were active genes, while only one or two genes were expressed in common wheat. This indicated that $T$. urartu is valuable in quality improvement in common wheat since a high number of active genes might contribute to superior bread-making quality [12]. Moreover, the i-type genes (A3-502/A3-573/A3-640, Glu-A3f) had positive effects on dough quality, e.g. percentage of SDS-unextractable fraction in total polymeric protein, dough resistance and extensibility [16]. The corresponding homologs of A3-573 and A3-640 were also detected in T. urartu.

\section{Center of origin and diversity of $T$. urartu}

Turkey was established as the center of origin and diversity with abundant plant species and endemism based on its variety in geomorphology, topography and climate [35]. Furthermore, southeastern Turkey exhibits great genetic diversity of plants in the Triticeae family, and is supposed to be the origin of domestication for wheat and einkorn (T. monococcum) [36]. Among the six collection areas in this work, southeastern Turkey showed the highest genetic diversity of LMW-GS genes (35 of the total 39 variants) and genotypes (13 of the total 15 genotypes) in $T$. 
urartu (Figures 3b, 4a; Table 2). Almost all of the genes/ variants and genotypes detected in the remaining areas were also detected in southeastern Turkey. Moreover, many variants (e.g., TuA3-463, TuA3-498, TuA3-535, TuA3-576c) and genotypes (U1, U3, U4, U5, U11, U12 and U14) were unique to southeastern Turkey (Figures 3b, 4a; Table 2). Even though the U8 genotype was exclusively detected in northeastern Lebanon and Syria and the U15 genotype was specifically identified in Armenia, similar genotypes were widely present in southeastern Turkey (U8 with U10 and U15 with U2 and U7) (Table 2). Considering the largest genetic diversity and typical LMW-GS genes/variants and genotypes, southeastern Turkey might be the center of origin and diversity of T. urartu. This conclusion was confirmed by the analysis of the loci coding storage proteins $[29,37]$ and the assessment of AFLP markers [25].

Lebanon was supposed as a center of specific adaptation for diploid and tetraploid wheats given that some morphological characters were exclusively detected there [38]. However, fewer unique LMW-GS genes/variants and genotypes were detected in T. urartu accessions from northeastern Lebanon than those from southeastern Turkey (Figures 3b, 4a; Table 2). Northwestern Syria was regarded as one of the regions of richest genetic diversity of $T$. urartu based on the assessment by AFLP markers [25]. Iran is one of the primary centers of diversity for wheat and its relatives; wild wheats, in particular diploid species, are extensively distributed in its various parts [39]. However, low genetic diversity of $T$. urartu in Syria and Iran was detected due to the lack of accessions collected, and the LMW-GS genes/variants and genotypes identified in these two areas were shared by southeastern Turkey and/or northeastern Lebanon (Figures 3b, 4a; Table 2). Larger collections of $T$. urartu are needed for further analyses to draw more precise conclusions about the diversity of LMW-GS genes/variants and genotypes in Syria and Iran.

\section{Direct A genome donors of T. aestivum}

Common wheat (AABBDD) is believed to be the result of spontaneous crosses between $T$. dicoccoides $\left(\mathrm{A}^{\mathrm{u}} \mathrm{A}^{\mathrm{u}} \mathrm{BB}\right)$ and Ae. tauschii (DD); $T$. dicoccoides $\left(\mathrm{A}^{\mathrm{u}} \mathrm{A}^{\mathrm{u}} \mathrm{BB}\right)$ was produced by the hybridization between $T$. urartu $\left(\mathrm{A}^{\mathrm{u}} \mathrm{A}^{\mathrm{u}}\right)$ and the $\mathrm{B}$ genome ancestor which was speculated as Ae. speltoides (SS) [2]. Considering its wide adaptability and variation, common wheat is believed to have arisen more than once from crosses of different genotypes of its progenitor species $[40,41]$. The determination of the specific donors of the A genome of bread wheat would benefit not only the genetic diversity conservation of $T$. urartu but expand the genetic basis for bread wheat breeding. The dissection of the LMW-GS gene family certainly would provide some evidence about the direct donors of the A genome of common wheat.
T. urartu and common wheat shared two genes, $A 3-$ 391 and $A 3-400$ (Figure 5a). The allelic variants for each gene showed high identity (>97\%), thus it was difficult to match the allelic variants between $T$. urartu and common wheat. The other two genes, TuA3-385 and TuA3-460 were unique to $T$. urartu (Figure $5 \mathrm{a}$ ). The itype genes were present as haplotypes and showed high diversity in common wheat and T. urartu. Except A3502 shared by $T$. urartu and common wheat (Figure $5 \mathrm{~b}$ ), the other i-type genes in common wheat were divided into five groups, from $\mathrm{i}_{\mathrm{A}}-1$ to $\mathrm{i}_{\mathrm{A}}-5$ [17], of which $\mathrm{i}_{\mathrm{A}}-3$ (A3-573/A3-640) and $\mathrm{i}_{\mathrm{A}}-4$ (A3-649-1/A3-649-2) contained the same number of i-type genes with $T$. urartu (Figure 5b). The TuA3-538 genes showed close relationship with $A 3-640\left(\mathrm{i}_{\mathrm{A}}-3\right)$ and A3-649-2 $\left(\mathrm{i}_{\mathrm{A}}-4\right)$, and the TuA3-576 genes showed higher identity with $A 3-573$ $\left(\mathrm{i}_{\mathrm{A}}-3\right)$ and A3-649-1 $\left(\mathrm{i}_{\mathrm{A}}-4\right)$ than the other genes (Figure $5 \mathrm{~b}$ ). Thus, the characterized $T$. urartu might be the direct donor of the Glu-A3 locus of common wheat varieties possessing $i$-type genes $i_{A}-3$ and $i_{A}-4$. Moreover, the $\mathrm{i}$-type genes $\mathrm{i}_{\mathrm{A}}-3$ and $\mathrm{i}_{\mathrm{A}}-4$ should be the ancient genotypes because they had the same number of i-type genes with $T$. urartu and their genes closely matched those in T. urartu with high identity (Figure 5b) [17]. Interestingly, group $\mathrm{i}_{\mathrm{A}}-4$ were only detected from landraces in the micro-collection of Chinese wheat germplasm, which also suggested that $\mathrm{i}_{\mathrm{A}}-4$ might be an ancient genotype [17]. The $\mathrm{i}_{\mathrm{A}}-1$ and $\mathrm{i}_{\mathrm{A}}-2$ groups might also be derived from the characterized $T$. urartu because their i-type genes shared the same branch with TuA3-576. But $\mathrm{i}_{\mathrm{A}}-1$ and $\mathrm{i}_{\mathrm{A}}-2$ groups only contained one i-type genes, which shared higher identity with TuA3-576 than TuA3-538 (Figure 5b). Thus, in these genotypes, TuA3-538 was lost due to a deletion and many SNP mutations of TuA3-576 were introduced during polyploidization. The $\mathrm{i}_{\mathrm{A}}-5$ was a special group of $\mathrm{i}$-type genes because all three genes were substantially different from the i-type genes in T. urartu (Figure 5b). This group of i-type genes in common wheat might be derived from some other LMW-GS genotypes not detected in the present study, or they might have undergone many deletion and duplication processes during their evolution.

\section{Conclusions}

In summary, this work has promoted our understanding of the composition, variation, expression and evolution of LMW-GS genes in T. urartu. Analysis of the geographic distribution of LMW-GS genes/variants and genotypes would facilitate the in situ conservation of the genetic diversity of $T$. urartu. These new LMW-GS genes/variants would broaden the genetic resources in wheat quality breeding and accelerate their application to improve bread-making quality in common wheat. 


\section{Methods}

\section{T. urartu accessions}

The T. urartu accessions were obtained from the State Key Laboratory of Plant Cell and Chromosome Engineering, the Institute of Genetics and Developmental Biology, and the Chinese Academy of Sciences. This collection consisted of 157 accessions including 82 from northeastern Lebanon (Iaat, Kfardane, Talia and Baalbek), 63 from southeastern Turkey (Mardin and Urfa), five from Armenia, five from Syria (Damascus and Haseke), one from Iraq (Arbil) and one from Iran (Bakhtaran) (Additional file 1: Table S1).

\section{DNA isolation and polymerase chain reaction (PCR) amplification}

Genomic DNA of 157 T. urartu accessions were extracted from young leaves of 14-day-old seedlings grown in a glasshouse using the cetyltrimethyl ammonium bromide (CTAB) method [42]. PCR was conducted using 20- $\mu \mathrm{l}$ reaction volumes consisting 1.0 U LA Taq DNA polymerase (Takara Bio, Otsu, Japan), $1 \times$ GC buffer I $\left(\mathrm{Mg}^{2+}\right.$, plus), 8 $\mathrm{nM}$ of each dNTP, $100 \mathrm{ng}$ of genomic DNA and 6 pmol of each specific primer. The PCR reactions were performed using a Veriti Thermal Cycler (Applied Biosystems, Foster City, CA, USA) according to Zhang et al. [13].

\section{Detection of LMW-GS genes}

LMW-GS genes were detected using the LMW-GS gene molecular marker system [13]. PCR products were purified with 3.0 M sodium acetate and 70\% ethanol before adding HiDi-formamide and GeneScan 1200 LIZ size standard (Applied Biosystems, Foster City, CA, USA). DNA fragments of LMW-GS genes were separated by capillary electrophoresis using a 3730xl DNA Analyzer (Applied Biosystems, Foster City, CA, USA) with the default genotyping module and the G5 dye set. LMW-GS genes and allelic variants were designated in accordance with the size of their corresponding fragment lengths in the GeneMapper Software v3.7 (e.g., 385 and 397) [13].

\section{Cloning of LMW-GS genes}

Fifty accessions were chosen to clone LMW-GS genes whose DNA fragment lengths were detected by the marker system [15]. Genes were cloned using the full length gene method and were further nominated as per the above cloning method [15]. Briefly, those sequences with high identity but a different length of repetitive domains were assigned to a single gene. Conversely, in a single gene, those sequences of conserved SNPs or different fragment lengths were considered allelic variants of the gene. Each gene was represented by the variant detected in the majority of accessions and designated as 'representative variant DNA fragment length + gene'. Similarly, allelic variants were named, 'DNA fragment length + allele', and letters in alphabetical order were added to distinguish these variants with the same fragment lengths but different SNPs according to their frequencies in the $T$. urartu population. For example, considering their high identity (>99\%), TuA3-460, TuA3463 and TuA3-474 were regarded as allelic variants of the gene TuA3-460; for TuA3-460 was detected in the majority of accessions (21), whereas TuA3-463 and TuA3-474 were only in three and four accessions, respectively (Additional file 2: Figure S8).

\section{Analysis of LMW-GS gene sequences}

The assembly and alignment of LMW-GS gene sequences were performed with the Lasergene software (DNASTAR; http://www.dnastar.com/). Sequence alignment results were visualized with GeneDoc (http://www.nrbsc.org/gfx/ genedoc/ebinet.htm). The phylogenetic trees of DNA sequences or predicted protein sequences of LMW-GS genes were constructed using the ClustalW2 (http://www. ebi.ac.uk/Tools/msa/clustalw2) and MEGA5 software [43] with the Neighbor-joining method.

\section{Separation and characterization of LMW-GSs}

To elucidate the expression pattern of LMW-GS genes in T. urartu, four accessions, which in turn contained one (PI428202), two (PI428255), three (PI428270) and four (PI428335) LMW-GS genes with intact ORFs, were chosen for proteomic analysis. In each accession, glutenins were extracted from three seeds with their embryos removed [44]. Then, the prepared glutenin samples were separated by 2-DE [12], and all the spots on 2-DE gels of PI428202, PI428255 and PI428270, were digested by chymotrypsin (Sigma-Aldrich, MO, USA) and identified by LC-MS/MS $[12,45]$. The LC-MS/MS spectra were analyzed with Bioworks 3.1 software, using a database including protein sequences of Triticeae available in NCBI (before 2013-7), deduced amino acid sequences from the $T$. urartu genomic data (http://gigadb.org/dataset/100050) and protein sequences of LMW-GS genes cloned in this work. The unidentified spots were further analyzed using the MALDI-TOF/TOF mass spectrometry (AB SCIEX 5800). MS and MS/MS data were analyzed using MASCOT 2.0 search engine (Matrix Science, London, U.K.) to search against the same database of the former LC-MS/MS, with the peptide mass tolerance and the MS/MS ion tolerance of $0.2 \mathrm{Da}$ and $0.5 \mathrm{Da}$, respectively. The protein scores greater than 58 were significant $(\mathrm{p}<0.05)$. Considering the identical electrophoretic mobility of the above three accessions, only the spots of LMWGSs in PI428335 were selected for mass spectra analysis. After verifying its consistency with 2-DE, SDS-PAGE was exploited to separate the LMW-GSs of every T. urartu accession for its high efficiency. 
Availability of supporting data

The resulting 148 LMW-GS sequences data were deposited in GenBank (http://www.ncbi.nlm.nih.gov) under the accessions of KM065455-KM065457 and KM085178KM085322. Other supporting data, Additional file 2.pdf and Additional file 1.pdf, are included as additional files of this manuscript.

\section{Additional files}

Additional file 1: Table S1. Collection site of the 157 T. urartu accessions. Table S2. Composition and variation of LMW-GS genes in T. urartu population. Table S3. Matching LMW-GS protein spots detected by 2-DE to the proteins predicted from the cloned active LMW-GS genes in T. urartu based on their Mass spectra. Table S4. LC-MS/MS identification of the protein spots on 2-DE gels of the glutenin fraction from $T$. urartu accessions. Table S5. MOLDI-TOF/TOF identification of the protein spots on 2-DE gels of the glutenin fraction from T. urartu accessions. Table S6. Nucleotide sequence identities of LMW-GS genes from $T$. urartu to the previously reported genes/allelic variants.

Additional file 2: Figure S1. Sequence alignments of the TUA3-385 gene identified in T. urartu and its homologs in common wheat and Ae. tauschii. Figure S2. Sequence alignments of the TuA3-391 gene identified in T. urartu. Figure S3. Sequence alignments of the TuA3-397 and TuA3-400 genes identified in T. urartu. Figure S4. Sequence alignments of the TuA3-502 gene identified in T. urartu. Figure S5. Sequence alignments of the TuA3-538 gene identified in T. urartu. Figure S6. Sequence alignments of the TuA3-576 gene identified in T. urartu. Figure S7. Protein sequence alignments of variants of TuA3-460 gene with s- and m-type genes characterized previously at the Glu-B3 and Glu-D3 loci in common wheat and $\mathrm{m}$-type genes in T. urartu. Figure $\mathbf{S 8}$. Sequence alignments of the TuA3-460 gene identified in T. urartu. Figure S9. Sequence alignments of the deduced proteins of all active LMW-GS genes in T. urartu.

\section{Abbreviations \\ 2-DE: Two-dimensional electrophoresis; CTAB: Cetyltrimethyl ammonium bromide; HMW-GS: High-molecular-weight glutenin subunit; LC-MS/MS: Liquid chromatography tandem mass spectrometry; LMW-GS: Low-molecular-weight glutenin subunit; MALDI-TOF/TOF-MS: Matrix assisted laser desorption/ ionization time of flight tandem mass spectrometry; MCC: Micro-core collections; ORF: Open reading frame; PCR: Polymerase chain reaction; pl: Isoelectric point; SDS-PAGE: Sodium dodecyl sulphate polyacrylamide gel electrophoresis.}

\section{Competing interests}

The authors declare that they have no competing interests.

\section{Authors' contributions}

GL carried out most experiments and wrote the manuscript. XZ analyzed the data and revised the manuscript. YZ cloned the LMW-GS genes. WY performed 2-DE. JS and YL provided and multiplied T. urartu lines. KZ critically revised the manuscript. AM and DL conceptualized the experiments and revised the manuscript. All authors read and approved the final manuscript.

\section{Acknowledgments}

The authors are grateful to M. Kathryn Turner, Department of Agronomy and Plant Genetics, University of Minnesota, for reviewing this manuscript. This work was supported by the National Science Foundation of China (31371610), the National Key Basic Research Program of China (2014CB138100) and the Ministry of Agriculture of China for transgenic research (2014ZX08009-003).

\section{Author details}

'State Key Laboratory of Plant Cell and Chromosome Engineering, National Center for Plant Gene Research, Institute of Genetics and Developmental Biology, Chinese Academy of Sciences, 1 West Beichen Road, Chaoyang District, Beijing 100101, China. ${ }^{2}$ University of Chinese Academy of Sciences, Beijing 100049, China. ${ }^{3}$ College of Agronomy, The Collaborative Innovation
Center of Grain Crops in Henan, Henan Agricultural University, 63 Nongye Road, Zhengzhou 450002, China. ${ }^{4}$ Present address: Department of Agronomy \& Plant Genetics, University of Minnesota, 1991 Buford Circle, St. Paul, MN 55108, USA.

Received: 25 July 2014 Accepted: 6 November 2014 Published online: 28 February 2015

\section{References}

1. Shewry PR, Tatham AS, Barro F, Barcelo P, Lazzeri P. Biotechnology of breadmaking: unraveling and manipulating the multi-Protein gluten complex. Biotechnology. 1995;13(11):1185-90.

2. Shewry PR. Wheat. J Exp Bot. 2009;60(6):1537-53.

3. D'Ovidio R, Masci S. The low-molecular-weight glutenin subunits of wheat gluten. J Cereal Sci. 2004;39(3):321-39.

4. Juhász A, Gianibelli M. Low-molecular-weight glutenin subunits: insight into this abundant subunit group present in glutenin polymers, Gliadin and glutenin: the unique balance of wheat quality. St Paul: AACC International Press; 2006. p. 171-212.

5. Ciaffi M, Tozzi L, Borghi B, Corbellini M, Lafiandra D. Effect of heat shock during grain filling on the gluten protein composition of bread wheat. J Cereal Sci. 1996;24(2):91-100.

6. Payne PI. Genetics of wheat storage proteins and the effect of allelic variation on bread-making quality. Annu Rev Plant Phys. 1987;38:141-53.

7. Gupta RB, Bekes F, Wrigley CW. Prediction of physical dough properties from glutenin subunit composition in bread Wheats: correlation studies. Cereal Chem. 1991;68(4):328-33.

8. Lew EJ-L, Kuzmicky D, Kasarda DD. Characterization of low molecular weight glutenin subunits by reversed-phase high-performance liquid chromatography, sodium dodecyl sulfate-polyacrylamide gel electrophoresis, and N-terminal amino acid sequencing. Cereal Chem. 1992;69:508-508.

9. Jackson EA, Holt LM, Payne PI. Characterization of high molecular-weight gliadin and low-molecular-weight glutenin subunits of wheat endosperm by two-dimensional electrophoresis and the chromosomal localization of their controlling genes. Theor Appl Genet. 1983;66(1):29-37.

10. Ikeda TM, Nagamine T, Fukuoka H, Yano H. Identification of new low-molecular-weight glutenin subunit genes in wheat. Theor Appl Genet. 2002;104(4):680-7.

11. Huang $\mathrm{X}-\mathrm{Q}$, Cloutier S. Molecular characterization and genomic organization of low molecular weight glutenin subunit genes at the Glu-3 loci in hexaploid wheat (Triticum aestivum L.). Theor Appl Genet. 2008;116(7):953-66.

12. Dong LL, Zhang XF, Liu DC, Fan HJ, Sun JZ, Zhang ZJ, et al. New insights into the organization, recombination, expression and functional mechanism of low molecular weight glutenin subunit genes in bread wheat. PLos One. 2010;5(10):e13548.

13. Zhang XF, Liu DC, Yang WL, Liu KF, Sun JZ, Guo XL, et al. Development of a new marker system for identifying the complex members of the lowmolecular-weight glutenin subunit gene family in bread wheat (Triticum aestivum L.). Theor Appl Genet. 2011;122(8):1503-16.

14. Espí A, Rodríguez-Quijano M, Vázquez JF, Carrillo JM, Giraldo P. Molecular characterization of Glu-B3 locus in wheat cultivars and segregating populations. J Cereal Sci. 2014;60(2):374-81.

15. Zhang XF, Liu DC, Jiang W, Guo XL, Yang WL, Sun JZ, et al. PCR-based isolation and identification of full-length low-molecular-weight glutenin subunit genes in bread wheat (Triticum aestivum L.). Theor Appl Genet. 2011;123(8):1293-305.

16. Zhang XF, Jin H, Zhang Y, Liu DC, Li GY, Xia XC, et al. Composition and functional analysis of low-molecular-weight glutenin alleles with Aroona near-isogenic lines of bread wheat. BMC Plant Biol. 2012;12:243.

17. Zhang XF, Liu DC, Zhang JH, Jiang W, Luo GB, Yang WL, et al. Novel insights into the composition, variation, organization, and expression of the low-molecular-weight glutenin subunit gene family in common wheat. J Exp Bot. 2013;64(7):2027-40

18. Peng JH, Sun D, Nevo E. Domestication evolution, genetics and genomics in wheat. Mol Breed. 2011;28(3):281-301.

19. Baum M, Appels R. Review: the cytogenetic and molecular architecture of chromosome $1 R$ - one of the most widely utilized sources of alien chromatin in wheat varieties. Chromosoma. 1991;101(1):1-10.

20. Cox T, Raupp W, Gill B. Leaf rust-resistance genes $L r 41$, $L r 42$, and $L r 43$ transferred from Triticum tauschii to common wheat. Crop Sci. 1994; 34(2):339-43. 
21. Joppa L, Du C, Hart GE, Hareland GA. Mapping gene (s) for grain protein in tetraploid wheat (Triticum turgidum L.) using a population of recombinant inbred chromosome lines. Crop Sci. 1997;37(5):1586-9.

22. Zhao $\mathrm{CH}$, Cui $F$, Wang $X Q$, Shan SC, Li XF, Bao YG, et al. Effects of $1 B L / 1 R S$ translocation in wheat on agronomic performance and quality characteristics. Field Crop Res. 2012;127:79-84.

23. Takumi S, Nasuda S, Liu YG, Tsunewaki K. Wheat phylogeny determined by RFLP analysis of nuclear DNA .1. Einkorn wheat. Jpn J Genet. 1993:68(1):73-9.

24. Vierling RA, Nguyen HT. Use of RAPD markers to determine the genetic diversity of diploid, wheat genotypes. Theor Appl Genet. 1992;84(7-8):835-8.

25. Baum BR, Bailey LG. Genetic diversity in the Red wild einkorn: T. urartu Gandilyan (Poaceae: Triticeae). Genet Resour Crop Ev. 2013;60(1):77-87.

26. Qiu Y, Zhou R, Kong X, Zhang S, Jia J. Microsatellite mapping of a Triticum urartu Tum. derived powdery mildew resistance gene transferred to common wheat (Triticum aestivum L.). Theor Appl Genet. 2005;111(8):1524-31.

27. Rouse M, Jin Y. Stem rust resistance in A-genome diploid relatives of wheat. Plant Dis. 2011:95(8):941-4.

28. Ling H-Q, Zhao S, Liu D, Wang J, Sun H, Zhang C, et al. Draft genome of the wheat A-genome progenitor Triticum urartu. Nature. 2013:496(7443):87-90.

29. Caballero L, Martin MA, Alvarez JB. Genetic diversity for seed storage proteins in Lebanon and Turkey populations of wild diploid wheat (Triticum urartu Thum. ex Gandil.). Genet Resour Crop Ev. 2009;56(8):1117-24.

30. Alvarez JB, Gutiérrez MV, Guzmán C, Martín LM. Molecular characterisation of the amino- and carboxyl-domains in different Glu-A1x alleles of Triticum urartu Thum. ex Gandil. Theor Appl Genet. 2013:126(7):1703-11.

31. Long H, Huang Z, Wei YM, Yan ZH, Ma ZC, Zheng YL. Length variation of i-type low-molecular-weight glutenin subunit genes in diploid wheats. Russ J Genet. 2008;44(4):429-35.

32. Moghaddam M, Ehdaie B, Waines JG. Genetic diversity in populations of wild diploid wheat Triticum urartu Tum. ex. Gandil. revealed by isozyme markers. Genet Resour Crop Ev. 2000;47(3):323-34.

33. Zohary D, Hopf M. Domestication of plants in the world. The origin and spread of cultivated plants in West Asia, Europe and the Nile valley. Oxford: Clarendon; 1993

34. Masci S, D'Ovidio R, Lafiandra D, Kasarda DD. Characterization of a low-molecular-weight glutenin subunit gene from bread wheat and the corresponding protein that represents a major subunit of the glutenin polymer. Plant Physiol. 1998:118(4):1147-58.

35. Karagoz A. Plant genetic resources conservation in Turkey. In: Duzyaman E, Tuzel Y, editors. Proceedings of the International Symposium on Sustainable Use of Plant Biodiversity to Promote New Opportunities for Horticultural Production Development. 2003. p. 17-25.

36. Dvorak J, Luo MC, Akhunov ED. NI Vavilov's theory of centres of diversity in the light of current understanding of wheat diversity, domestication and evolution. Czech J Genet Plant. 2011:47:S20-7.

37. Caballero L, Martin MA, Alvarez JB. Allelic variation for the high- and lowmolecular-weight glutenin subunits in wild diploid wheat (Triticum urartu) and its comparison with durum wheats. Aust J Agr Res. 2008;59(10):906-10

38. Hammer K, Filatenko AA, Korzun V. Microsatellite markers - a new tool for distinguishing diploid wheat species. Genet Resour Crop Ev. 2000;47(5):497-505.

39. Salimi A, Ebrahimzadeh H, Taeb M. Description of Iranian diploid wheat resources. Genet Resour Crop Ev. 2005:52(4):351-61.

40. Kuckock V. Experimental investigation on the origin of wheat. Zeitschr Pfl Züchtung. 1964;51:97-140.

41. Talbert L, Smith L, Blake N. More than one origin of hexaploid wheat is indicated by sequence comparison of low-copy DNA. Genome. 1998:41(3):402-7.

42. Saghaimaroof MA, Soliman KM, Jorgensen RA, Allard RW. Ribosomal DNA spacer-length polymorphisms in barley: Mendelian inheritance, chromosomal location, and population dynamics. P Natl Acad Sci-Biol. 1984:81(24):8014-8.

43. Kumar S, Nei M, Dudley J, Tamura K. MEGA: A biologist-centric software for evolutionary analysis of DNA and protein sequences. Brief Bioinform. 2008;9(4):299-306.

44. Singh M, Chabane K, Valkoun J, Blake T. Optimum sample size for estimating gene diversity in wild wheat using AFLP markers. Genet Resour Crop Ev. 2006;53(1):23-33.

45. Ge P, Ma C, Wang S, Gao L, Li X, Guo G, et al. Comparative proteomic analysis of grain development in two spring wheat varieties under drought stress. Anal Bioanal Chem. 2012:402(3):1297-313.

\section{Submit your next manuscript to BioMed Central and take full advantage of:}

- Convenient online submission

- Thorough peer review

- No space constraints or color figure charges

- Immediate publication on acceptance

- Inclusion in PubMed, CAS, Scopus and Google Scholar

- Research which is freely available for redistribution

Submit your manuscript at www.biomedcentral.com/submit 